\title{
Research on the Gauge-Invariant Energy Density of Similar Electromagnetic Properties of Gravitational Field
}

\author{
Benchao Zhu ${ }^{1, a}$, Weiwei Jiang ${ }^{2, b^{*}}$ and Fan Zhang ${ }^{2, c}$ \\ ${ }^{1}$ Department of physics and mathematics, Hubei University of Medicine, shiyan, China \\ ${ }^{2}$ Hubei Dongfeng Automobile Technician College, Shiyan 442000, China \\ azbc@hbmu.edu.cn, bjww0507@163.com, czhangfan12@163.com
}

Keywords: Gauge-invariant; Energy density; Similar electromagnetic; Gravitational field

\begin{abstract}
This paper will focus on the relation between gauge-invariant energy density of gravitational field and electromagnetic field. After the brief review the history of finding the analogy between gravitation and electromagnetism, we recall the two different ways to remove the gauge degrees of freedom, Physical decomposition and gauge-fixing. Then, by using the gauge-fixing approach, we get the gauge-invariant energy density (Hamilton density) of gravitational field. By using the similar method into electrodynamics, we can also get the gauge-invariant energy density of electromagnetic field. Finally, while comparing with the two gauge-invariant energy density of gauge-invariant energy density of gravitational field and electromagnetic field, we find there is a similar electromagnetic energy density does appear in gravitational field. The amazing conclusion is that although the rotating electric charge with constant speed can produce static magnetic field energy, the rotating mass with constant speed cannot produce any similar "magnetic" energy in gravitational field. Only accelerated rotating mass can produce the similar "magnetic" energy, this is a peculiar property of gravitational field never reported before.
\end{abstract}

\section{Introduction}

Field theories form the present theoretical framework of our understanding the fundamental interactions of nature. In the quantum field theory, one interaction corresponds to a special field. The history of research the relation among the various interactions can be dated very early. The similarity between Newton's law of gravitation and Coulomb's law of electricity naturally led to a gravitoelectric description of Newtonian gravitation. Then, in the middle of the 19th Century, Maxwell's electromagnetic field theory unified the function of electricity and magnetism. It was the first unified theory of several interactions in history. At the beginning of 20 Century, Einstein had broken Newton's concept of the super distance in the theory of gravity, and introduced the theory of the field of gravity to create the theory of general relativity. After then, the enthusiasm research about unifying the gravitational field and electromagnetic field blow out. To some extent, it's because of Einstein believed that the nature has only one force, others forces are just different forms of the unify field force, and Einstein devoted his later half life to unify the gravitational field and electromagnetic field [1]. Now, we know that, since there are totally four fundamental fields, all attempts to only unify the electromagnetic field and the gravitational field cannot be successful. But the research on the similar relation between gravitational field and electromagnetic field are still continued.

In this paper, we will present the relation of energy density between gravitational field and electromagnetic field. As we know that static charge can produce static electric field, static mass (we can call it static gravity "charge") also can produce static gravitational field. How about the moving electric charge and gravity charge? One can use the corresponding energy-momentum tensor of electromagnetic field and gravitational field to solve this problem. Unfortunately, the energy-momentum tensor of gravitational field is still an unsolved problem for 100 years. Only recent years, we revisited this old problem through the physical decomposition of gravitational field [2]. Only after solving the gauge-dependent problem of energy density of gravitational field, we can do 
attempt to research the energy density relation between moving electric charge and moving gravity "charge".

\section{Physical Decomposition and Gauge-fixing Condition of Gravitational Field}

Gauge dependence of the gravitational energy originates exactly from the fact that the metric may contain a spurious gravitational effect. In Ref. [2], the metric tensor $g_{\mu v}$ is unambiguously decomposed into the sum of a physical term $\hat{g}_{\mu v}$, which represents the true gravitational effect, and a pure geometric term $\bar{g}_{\mu \nu}$, which represents the spurious gravitational effect associated with coordinate choice. Affine connection is also decomposed into physical part and pure geometric part: $\Gamma_{\mu \nu}^{\rho}=\hat{\Gamma}_{\mu \nu}^{\rho}+\bar{\Gamma}_{\mu \nu}^{\rho}$. The relation between $\bar{\Gamma}_{\mu \nu}^{\rho}$ and $\bar{g}_{\mu \nu}$ is the same as whole $\Gamma_{\mu \nu}^{\rho}$ and $g_{\mu \nu}$ (Notation: Throughout this paper, an over dot denotes time derivative, Greek indices run from 0 to 3, Latin indices run from 1 to 3 , and repeated indices are summed over even when two spatial indices are both upstairs or downstairs, $\partial$ or comma denotes ordinary derivative.)

$$
\bar{\Gamma}_{\mu \nu}^{\rho}=\frac{1}{2} \bar{g}^{\rho \sigma}\left(\partial_{\mu} \bar{g}_{v \sigma}+\partial_{\nu} \bar{g}_{\mu \sigma}-\partial_{\sigma} \bar{g}_{\mu v}\right)
$$

Here $\bar{g}^{\mu \nu}$ is defined as the inverse of $\bar{g}_{\mu \nu}$, i.e., $\bar{g}^{\mu \rho} \bar{g}_{\rho v}=\delta_{v}^{\mu}$. But physical connection $\hat{\Gamma}_{\mu \nu}^{\rho}$ is not related to $\hat{g}_{\mu \nu}$ as in Eq. (1). Mathematically, a well-defined separation $g_{\mu \nu}=\hat{g}_{\mu \nu}+\bar{g}_{\mu \nu}$ means an unambiguous prescription for constructing $\hat{g}_{\mu v}$ and $\bar{g}_{\mu \nu}$ out of a given $g_{\mu v}$.

$$
\begin{aligned}
& \bar{R}_{\sigma \mu \nu}^{\rho}=\partial_{\mu} \bar{\Gamma}_{\mu \nu}^{\rho}-\partial_{\nu} \bar{\Gamma}_{\sigma \mu}^{\rho}+\bar{\Gamma}_{\alpha \mu}^{\rho} \bar{\Gamma}_{\sigma \nu}^{\alpha}-\bar{\Gamma}_{\alpha \nu}^{\rho} \bar{\Gamma}_{\sigma \mu}^{\alpha}=0 \\
& g^{i j} \hat{\Gamma}_{i j}^{\rho}=0
\end{aligned}
$$

Here $\bar{R}_{\sigma \mu \nu}^{\rho}$ in Eq. (1a) is the Riemann curvature of $\bar{g}_{\mu \nu}$. Eq. (2) can give a solution for $\hat{\Gamma}_{\mu \nu}^{\rho}$ and $\bar{\Gamma}_{\mu \nu}^{\rho}$ (and further for $\hat{g}_{\mu \nu}$ and $\bar{g}_{\mu \nu}$ by Eq. (1). For the sake of simplicity, in this paper, we are only interested in weak-field approximation, which means the ordinary metric tensor can be written as $g_{\mu \nu}=\eta_{\mu \nu}+h_{\mu v}$, here $\eta_{\mu v}$ presents Minkowski metric and $h_{\mu \nu} \ll 1$ presents weak curvature of space-time. The solution of $\hat{\Gamma}_{\mu \nu}^{\rho}$ and $\hat{h}_{\mu \nu}$ must rely on perturbative method. The detail formations are not needed here. In order to distinguish Eq. (2) from other decompositions of gravitational field, Eq. (2) is called CZ decomposition in Ref. [2].

In an accompanying paper [3], we discuss gauge-fixing approach and find it is another possible way to remove gravitational gauge-dependence degrees, one special gauge is presented

$$
\partial_{i} h_{i \rho}-\frac{1}{2} \partial_{\rho} h_{i i}=0
$$

No spurious gravitational effect can be alived in gauge condition Eq. (3). CZ decomposition is equivalent to gauge Eq. (4) in removing non-physical degrees of freedom of gravitational field [3]. In another paper [4] we extend the gauge Eq. (3) to so-called general transverse radiation gauge:

$$
\partial_{i} h_{i 0}+a \partial_{0} h_{i i}=0, \quad \partial_{i} h_{i j}+b \partial_{j} h_{i i}=0
$$

$b \neq-1$. We had proved that each choice of parameter a, b in Eq. (4) corresponds to one way of decomposition $h_{\mu v}$ into a gauge-invariant part plus a pure-gauge part. Some special choices of a, b are particularly interesting and be detail discussed in Ref. [4], like CZ ( $\left.a=b=-\frac{1}{2}\right), \operatorname{ADM}(b=0)$, York $\left(b=-\frac{1}{3}\right)$, and Weinberg $\left(a=-\frac{2}{3}, b=-\frac{1}{3}\right)$. The final gauge-invariant solution of $h_{\mu v}$ solved by Einstein equation is 


$$
\begin{aligned}
& \hat{h}_{00}^{(a b)}=h_{00}-\bar{h}_{00}^{(a b)}=h_{00}-\frac{1}{\vec{\partial}^{2}}\left(2 h_{k 0, k 0}-h_{k k, 00}\right)-\frac{1+2 a}{1+b} \frac{1}{\vec{\partial}^{2}}\left(h_{k k}-\frac{1}{\vec{\partial}^{2}} h_{k l, k l}\right)_{, 00} \\
& \hat{h}_{0 j}^{(a b)}=h_{0 j}-\bar{h}_{0 j}^{(a b)}=h_{0 j}-\frac{1}{\vec{\partial}^{2}}\left(h_{k 0, k j}+h_{k j, k 0}-h_{k k, 0 j}\right)-\frac{1+a+b}{1+b} \frac{1}{\vec{\partial}^{2}}\left(h_{k k}-\frac{1}{\vec{\partial}^{2}} h_{k l, k l}\right)_{, 0 j} \\
& \hat{h}_{i j}^{(b)}=h_{i j}-\bar{h}_{i j}^{(a b)}=h_{i j}-\frac{1}{\vec{\partial}^{2}}\left(h_{k i, k j}+h_{k j, k i}-h_{k k, i j}\right)-\frac{1+2 b}{1+b} \frac{1}{\vec{\partial}^{2}}\left(h_{k k}-\frac{1}{\vec{\partial}^{2}} h_{k l, k l}\right)_{, i j}
\end{aligned}
$$

Superscript (ab) means gauge condition Eq. (4). It is easy to find Eq. (5) is equivalent to Eq. (2) when $a=b=-\frac{1}{2}$. The final solution of physical affine connection is

$$
\hat{\Gamma}_{\mu \nu}^{\rho(a b)}=\Gamma_{\mu \nu}^{\rho}-\bar{\Gamma}_{\mu \nu}^{\rho(a b)}=\Gamma_{\mu \nu}^{\rho}-\frac{1}{2}\left(\partial_{\mu} \bar{h}_{\nu}^{\rho(a b)}+\partial_{\nu} \bar{h}_{\mu}^{\rho(a b)}-\partial^{\rho} \bar{h}_{\mu \nu}^{(a b)}\right)
$$

$\bar{h}_{\mu v}^{(a b)}$ is non-physical metric solution in gauge condition Eq. (4), whose explicit expression is given in Eq. (5). Finally we have two different ways to remove gauge-dependence degrees of freedom, i.e., physical decomposition Eq. (1), and gauge-fixing approach Eq. (4) of gravitational field.

\section{Gauge-invariant Energy Density of Gravitational Field.}

There are two ways to get the energy density of gravitational field, one is to use the energy-momentum tensor of gravitational field, and the other one is to use the field theory of Hamilton density. As we had claimed in first section, till now, the energy-momentum tensor of gravitational field The Lagrangian density of gravitational field couples to an external conserved source $T^{\mu v}(x)$ which is invariant under gauge transformation $h_{\mu \nu}(x) \rightarrow h_{\mu \nu}^{\prime}(x)=h_{\mu \nu}(x)+\partial_{\mu} \xi_{v}(x)+\partial_{\nu} \xi_{\mu}(x)$ IS [5]:

$$
L=\frac{1}{4}\left(\partial_{\mu} h_{\alpha}^{\alpha} \partial^{\mu} h_{\beta}^{\beta}-\partial_{\mu} h_{\alpha \beta} \partial^{\mu} h^{\alpha \beta}+2 \partial_{\mu} h^{\mu \alpha} \partial^{v} h_{v \alpha}-2 \partial_{\mu} h_{\alpha}^{\alpha} \partial_{v} h^{\mu v}\right)+\frac{\kappa}{2} h_{\mu v} T^{\mu v}
$$

Given the Lagrangian, we can proceed to construct the canonical Hamilton of gravitational field

$$
H=\Pi_{\mu \nu} \dot{h}^{\mu \nu}-L
$$

Here $\frac{\partial L}{\partial \dot{h}^{\mu \nu}}=\Pi_{\mu \nu}$ is the momentum conjugate of $h^{\mu v}$. The momentum conjugates are

$$
\Pi_{00}=\frac{1}{2} \partial_{i} h_{0 i}, \Pi_{0 i}=\frac{1}{2}\left(\partial_{i} h_{00}-\partial_{i} h+2 \partial_{j} h_{i j}\right), \Pi_{i j}=\frac{1}{2}\left[\dot{h}_{i j}+\delta_{i j}\left(\partial_{k} h_{0 k}-\dot{h}\right)\right]
$$

$h \equiv h_{i i}$ is the spatial trace. (We have identified $\dot{h}_{0 i}$ with $\dot{h}_{i 0}$, but not $\dot{h}_{i j}$ with $\dot{h}_{j i}$, thus we are going to sum over both $(i j)$ and $(j i)$, but not $(i 0)$.). It is now straightforward to calculate the Hamilton quantity $\mathrm{H}$ : (Note that $\mathrm{H}$ is the Hamilton quantity and $H$ is the Hamiltonian density)

$$
\mathrm{H}=\int d^{3} x\left(\Pi_{\mu v} \dot{h}^{\mu v}-L\right)=\int d^{3} x\left(\Pi_{00} \dot{h}^{00}+\Pi_{i j} \dot{h}^{i j}+\Pi_{0 i} \dot{h}^{0 i}-L\right)
$$

When casting Eq. (4) and Eq.(5) into the equations for Eq. (9), the final gauge-invariant canonical Hamilton of GF can be written as following form

$$
\begin{aligned}
\mathrm{H}^{(a b)}= & \frac{1}{4} \int d^{3} x\left(\dot{h}_{i j}^{T T} \dot{h}_{i j}^{T T}+\partial_{i} h_{j k}^{T T} \partial_{i} h_{j k}^{T T}+2 h_{i j}^{T T} T_{i j}+\frac{1}{2} T_{00} \frac{1}{\overline{\partial^{2}}} T_{00}\right. \\
& \left.+T_{00} \frac{1}{\overline{\partial^{2}}} T_{k k}-2 T_{0 i} \frac{1}{\overline{\partial^{2}}} T_{0 i}+\frac{5 b-8 a-3}{2(1+b)} \frac{1}{\overrightarrow{\partial^{2}}} \dot{T}_{00} \frac{1}{\overrightarrow{\partial^{2}}} \dot{T}_{00}\right)
\end{aligned}
$$

Here superscript (ab) means the calculation are doing in general transverse gauge Eq. (4), and Eq. (11) is the gauge-invariant Hamilton quantity of gravitational field we want to get. 


\section{Gauge-invariant Energy Density of Electromagnetic Field.}

To understand better the canonical Hamilton of GF under "general transverse gauge" Eq. (4), it is helpful to recall the parallel procedure of canonical Hamilton of vector field (electrodynamics). Given the Lagrangian of vector gauge field $A^{\mu}$ couples to external electric fluid source $j^{\mu}$

$$
L^{A}=\frac{1}{2}\left(\partial_{\mu} A_{v} \partial^{v} A^{\mu}-\partial_{\mu} A_{v} \partial^{\mu} A^{v}\right)+e A_{\mu} j^{\mu}
$$

We can get the Hamilton quantity

$$
\mathrm{H}^{A}=\int d^{3} x\left\{\frac{1}{2}\left[\dot{A}_{j} \dot{A}_{j}+\partial_{i} A_{j}\left(\partial_{i} A_{j}-\partial_{j} A_{i}\right)-\partial_{i} A^{0} \partial_{i} A^{0}\right]-e A_{\mu} j^{\mu}\right\}
$$

The superscript $A$ denotes the vector gauge field. If we cast $\vec{A} \equiv \vec{A}_{\perp}+\vec{A}_{\|}, \vec{\partial} \cdot \vec{A}_{\perp}=0$ and $\vec{\partial} \times \vec{A}_{\|}=0$ into Eq. (12) (Coulomb gauge condition), the final pure physical Hamilton of vector field reads []

$$
\begin{aligned}
\mathrm{H}^{A} & =\frac{1}{2} \int d^{3} x\left[\dot{A}_{\perp}^{j} \dot{A}_{\perp}^{j}-\partial_{i} A^{0} \partial_{i} A^{0}-\partial_{i} A_{\perp}^{j}\left(\partial_{j} A_{\perp}^{i}-\partial_{i} A_{\perp}^{j}\right)+e J^{0} A^{0}-e \vec{j} ? \vec{A}_{\perp}\right. \\
& =\frac{1}{2} \int d^{3} x\left(\dot{\vec{A}}_{\perp}^{2}+\left(\vec{\partial} \times \vec{A}_{\perp}\right)^{2}+e J^{0} A^{0}-e \vec{j} ? \vec{A}_{\perp}\right)
\end{aligned}
$$

Eq. (14) tells us something already known, $\dot{A}_{\perp}^{j} \dot{A}_{\perp}^{j}$ denotes electromagnetic radiation energy density, $\frac{1}{2}\left(\vec{\partial} \times \vec{A}_{\perp}\right)^{2}$ is energy density of magnetic field $\frac{1}{2} \vec{B}^{2}$ which is generated by moving charged system, $\frac{1}{2} \int d^{3} x\left(e j^{0} A^{0}-e \vec{j} \cdot \vec{A}_{\perp}\right) \frac{1}{2} \int d^{3} x\left(e j^{0} A^{0}-e \vec{j} ? \vec{A}_{\perp} \quad\right.$ is the non-dynamical energy including Coulomb potential $e j^{0} A^{0}$ and reaction energy density (nonlinear effect) $\vec{j} \cdot \overrightarrow{A_{\perp}}$.

\section{Similar Static Magnetic Energy Density of Gravitational Field}

When comparing Eq. (11) with Eq. (15), we can find that $\partial_{i} h_{j k}^{T T} \partial_{i} h_{j k}^{T T}$ which is equivalent to $\left(\vec{\partial} \times \vec{A}_{\perp}\right)^{2}=\vec{B}^{2}$ denotes "magnetic" energy density. Thus we can explain it as similar "magnetic" energy density of gravitational field. Corresponding to a constant speed rotation charged system, which is steady ring electric current, will generate static magnetic field $\vec{B}$ in electrodynamics, one may expect $\partial_{i} h_{j k}^{T T} \partial_{i} h_{j k}^{T T}$ will generate similar " static magnetic" energy by a constant speed rotation gravitational source.

The outside metric tensor of constant speed rotating spherical mass under weak field approximation is first given by Lense and Thirring [6]

$$
d s^{2}=-\left(1-\frac{2 G M}{r}\right) d t^{2}+\left(1+\frac{2 G M}{r}\right) d \vec{x}^{2}-4 \frac{G J}{r^{3}}\left(x_{1} d x_{2} d t-x_{2} d x_{1} d t\right)
$$

Where $J$ and $M$ are respective moment of inertia and total mass of source. It is easy to check that Eq. (15) can be solved by field equation Eq. (5) when choosing $a=-\frac{1}{2}, b=-\frac{1}{3}$ in the general transverse gauge Eq. (4). When casting $b=-\frac{1}{3}$ in

$$
h_{i j}^{(a b)}=h_{i j}^{T T}+\frac{1+b}{2} \delta_{i j} h^{(a b)}-\frac{1+3 b}{2} \frac{1}{\vec{\partial}^{2}} \partial_{i} \partial_{j} h^{(a b)}
$$

We can get $h_{i j}^{T T}=0$. This result reveals something interesting and peculiar about gravitational field, since the constant speed rotation system can not generate dynamic energy density far away from the source. It means that although $\partial_{i} h_{j k}^{T T} \partial_{i} h_{j k}^{T T}$ is similar to $\left(\vec{\partial} \times \vec{A}_{\perp}\right)^{2}$ in mathematics, this similarity is just spurious and they are not equivalent each other in physics. As constant speed rotation charged system 
generates magnetic field $\vec{B}$, but constant speed rotation mass system doesn't generate any field like magnetic field $\vec{B}$ any more. It is an amazing conclusion about gravitational field never been discussed before. Although the similar static magnetic energy density of gravitational field is not existed, $\partial_{i} h_{j k}^{T T} \partial_{i} h_{j k}^{T T}$ may contribute some energy in radiation system is still an exciting conclusion.

\section{Summary}

In this paper we have carefully shown that, after solving the gauge-dependent problem of gravitational field by physical decomposition and gauge-fixing way, the gauge-invariant energy density (Hamilton density) of gravitational field can be reasonable constructed by our way. Comparing with the electromagnetic field, we find something interesting presented in the following Table 1 . The amazing conclusion is that although the rotating electric charge with constant speed can produce static magnetic field energy, the rotating mass with constant speed cannot produce any similar "magnetic" energy in gravitational field. Only accelerated rotating mass can produce the similar "magnetic" energy, and this peculiar property of gravitational field never reported before.

Table 1 Similar electromagnetic field energy of gravitational field

\begin{tabular}{|c|c|c|}
\hline Energy Form & Electromagnetic Field & Graivtational Field \\
\hline $\begin{array}{c}\text { Radiation Energy } \\
\text { Density }\end{array}$ & $\dot{A}_{\perp}^{j} \dot{A}_{\perp}^{j}$ & $\dot{h}_{i j}^{T T} \dot{h}_{i j}^{T T}$ \\
\hline $\begin{array}{c}\text { Energy Density of } \\
\text { "Magnetic Field" }\end{array}$ & $\frac{1}{2}\left(\vec{\partial} \times \vec{A}_{\perp}\right)^{2}$ & $\partial_{i} h_{j k}^{T T} \partial_{i} h_{j k}^{T T}$ \\
\hline $\begin{array}{c}\text { Non-Dynamical } \\
\text { Energy }\end{array}$ & $\frac{1}{2} \int d^{3} x\left(e j^{0} A^{0}-e j \cdot \vec{A}_{\perp}\right)$ & $\frac{1}{4} \int d^{3} x\left(2 h_{i j}^{T T} T_{i j}+\frac{1}{2} T_{00} \frac{1}{\overrightarrow{\partial^{2}}} T_{00}+T_{00} \frac{1}{\overrightarrow{\partial^{2}}} T_{k k}\right.$ \\
& $\left.-2 T_{0 i} \frac{1}{\vec{\partial}} T_{0 i}+\frac{5 b-8 a-3}{2(1+b)} \frac{1}{\overrightarrow{\partial^{2}}} \dot{T}_{00} \frac{1}{\overrightarrow{\partial^{2}}} \dot{T}_{00}\right)$ \\
\hline
\end{tabular}

\section{Acknowledgements}

This work is supported by the 2014 Hubei Education Department Scientific Project No. B2014058 and 2013 Hubei University of medicine graduate start-up fund N0. 2013QDJZR03.

\section{References}

[1] J Bahram Mashhoon, Gravitoelectromagnetism: A Brief Review. The Measurement of Gravitomagnetism: A Challenging Enterprise, edited by L. Iorio (Nova Science, New York, 2007), pp. 29-39.

[2] Xiang-Song Chen and Ben-Chao Zhu , Physical decomposition of the gauge and gravitational fields , Phys. Rev. D 83, 084006 (2011)

[3] Xiang-Song Chen and Ben-Chao Zhu, True radiation gauge for gravity, Phys. Rev. D 83, 061501(R) (2011)

[4] Ben-Chao Zhu and Xiang-Song Chen, Tensor gauge condition and tensor field decomposition, Mod. Phys. Lett. A 30, 1550192 (2015)

[5] Xiang-Song Chen, Ben-Chao Zhu, and Niall O MURCHADHA, Spin and Orbital Angular Momentum of the Tensor Gauge Field, J. Phys. Soc. Jpn. 82 ,083001 (2013)

[6] I J.Lense, H, Thirring, Phys. Z. 19, 156 (1918) 